\title{
Aspectos estruturais da cooperação entre pesquisadores no campo de administração pública e gestão social: análise das redes entre instituições no Brasil*
}

\author{
Luciano Rossoni** \\ Antônio João Hocayen-da-Silva*** \\ Israel Ferreira Júnior****
}

Sumário: 1. Introdução; 2. Abordagem de redes sociais; 3. Procedimentos metodológicos; 4. Discussão e análise dos resultados; 5. Considerações finais.

Summary: 1. Introduction; 2. Social networks approach; 3. Methodological procedures; 4. Discussion and analysis of the results; 5 . Final remarks.

Palavras-chave: análise de redes sociais; administração pública e gestão social; estrutura de relacionamento; produção científica; redes de pesquisa.

KEY WORDS: social network analysis; public sector management and social management; relationship structure; scientific production; research networks.

\footnotetext{
* Artigo recebido em ago. 2006 e aceito em abr. 2008. A versão preliminar deste artigo foi premiada como melhor trabalho do II Encontro de Administração Pública e Governança da Anpad (EnAPG 2006).

** Professor e pesquisador do Programa de Mestrado e Doutorado da Universidade Positivo (PMDA/UP). Doutorando e mestre em administração pelo Centro de Pesquisa e Pós-Graduação da Universidade Federal do Paraná (Ceppad/UFPR). Endereço: Rua Osmário de Lima, 598 - Capão da Imbuia — CEP 82810-260, Curitiba, PR, Brasil. E-mail: lrossoni@gmail.com.

*** Mestre em administração pelo Centro de Pesquisa e Pós-Graduação da Universidade Federal do Paraná (Ceppad/UFPR). Professor e coordenador acadêmico dos cursos de Gestão Financeira e de Marketing da Faculdade de Ensino Superior do Centro do Paraná (UCP). Endereço: Rua Maria Berger Grande, 470 - Centro - CEP 85200-000, Pitanga, PR, Brasil. E-mail: hocayen@ yahoo.com.br.

**** Doutorando e mestre em administração pelo Centro de Pesquisa e Pós-Graduação da Universidade Federal do Paraná (Ceppad/UFPR). Endereço: Rua Emílio Kuntz Busch, 343 — Jardim Anavec _ CEP 13485-157, Limeira, SP, Brasil. E-mail: isjunior99@yahoo.com.br.
} 
Este artigo verifica, por meio da análise de redes, a estrutura de relacionamento entre instituições de pesquisa no campo de administração pública e gestão social no Brasil. O relacionamento entre instituições de pesquisa foi obtido com base nas afiliações institucionais dos autores pela análise de 549 artigos publicados nos anais do Enanpad, entre 2000 e 2005. Com o uso dos softwares Ucinet 6.0 e Pajek 1.10, foi elaborada a rede de relações da área e foi possível verificar tanto as propriedades estruturais e posicionais da rede, quanto avaliar a influência da estrutura da rede na produção científica. Verificou-se que, apesar de a densidade da rede ser baixa, a distância entre as instituições é pequena, com grande componente principal. Podese afirmar que, na maioria dos casos, as instituições que apresentam maior número de laços estão mais globalmente centralizadas e também são importantes elos de coesão na rede. Observou-se que a maioria dos laços acontece entre instituições do próprio estado. Pode-se também afirmar que a estrutura da rede tem relação direta com os indicadores de produção científica.

Structural aspects of the cooperation among researchers in the field of public sector management and social management: analysis of institution networks in Brazil

This article examines, through network analysis, the relationship structure of research institutions in the field of public sector management and social management in Brazil. The relationship between research institutions is identified based on the institutional affiliations of the authors, by analyzing 549 papers published in the annals of the Anpad annual meeting, from 2000 to 2005. Aided by the Ucinet 6.0 and Pajek 1.10 software, the relationship network was established, making it possible to examine not only the structural and positional properties of the network, but also the influence of the network structure on the scientific production. It was found that, despite the network's low density, the distance between institutions is small, with a much larger central component. In most cases, it can be suggested that the institutions that have the higher number of loops are globally centered and are also important to the network's cohesion. Most loops occur between institutions in the same state. Moreover, the network structure is directly correlated with the production indicators.

\section{Introdução}

Há tempos, muitos pesquisadores vêm avaliando as características da produção científica na área da administração. Entretanto, tais estudos ainda não avaliaram a estrutura de colaboração entre as instituições de pesquisa no país. Partindo-se do pressuposto de que o conhecimento científico é construído socialmente, influenciado pelos pares que compõem estruturalmente a rede de relações entre as instituições, busca-se não somente descrever tais rela- 
cionamentos, mas também entender como tal estrutura afeta a produção do conhecimento. Depois verificou-se, por meio da análise de redes, a estrutura de relacionamento entre instituições de pesquisa no campo de administração pública e gestão social no Brasil.

Desse modo, partindo-se da experiência de avaliação no campo de ciência e tecnologia (Rossoni, Hocayen-da-Silva e Ferreira Júnior, 2008) e de outras áreas do conhecimento que utilizaram a análise de redes sociais para elucidar a estrutura de um campo científico (Barabasi et al., 2002; Guimera et al., 2005; Liu et al., 2005; Moody, 2004; Newman, 2001a, 2001b, 2001c, 2004; Wagner e Leydesdorff, 2005), pretendeu-se com este artigo preencher essa lacuna.

Caracteristicamente, as pesquisas em redes sociais buscam avaliar a estrutura de relacionamento entre atores sociais. Este artigo, particularmente, avalia a estrutura de relações entre as instituições a partir dos relacionamentos entre os pesquisadores do campo da administração pública e gestão social, que publicaram artigos científicos no Enanpad - Encontro Nacional da Associação Nacional de Pós-Graduação e Pesquisa em Administração, entre 2000 e 2005.

O artigo está estruturado em cinco seções. Após breve introdução, que apresenta o objetivo, são apresentados os conceitos teóricos relacionados à análise de redes sociais que nortearam a pesquisa. Posteriormente, são discutidos os procedimentos metodológicos. Por fim, têm-se os resultados e as considerações finais.

\section{Abordagem de redes sociais}

Emirbayer e Goodwin (1994) salientam que a análise de redes sociais não é uma teoria formal ou unitária, mas ampla estratégia de investigação de estruturas sociais. Nelson (1984) afirma que, em termos intuitivos, as redes sociais são conjuntos de contatos que ligam vários atores, nos quais tais contatos podem ser de diferentes tipos, apresentarem conteúdos distintos, bem como diversas propriedades estruturais. A análise de redes sociais é inerentemente de natureza interdisciplinar, possuindo contribuições de áreas como matemática, estatística e computação, no ímpeto de produzir aplicações para o método (Freeman, 1984).

Existe uma série de conceitos-chave essenciais para a discussão da análise de redes sociais, que são (Wasserman e Faust, 1994): 
v ator - são as entidades (indivíduos, organizações ou países) objetos de estudo na análise de redes sociais;

v laço relacional - definido como a ligação estabelecida entre o par de atores;

v díade - ligação ou relacionamento estabelecido entre dois atores;

v tríade - conjunto de três atores e os possíveis laços entre eles;

v subgrupo - conjunto de atores e todos os laços entre eles;

v grupo - finito conjunto de atores definidos por critérios conceituais, teóricos ou empíricos, em que as medidas da rede são tomadas;

v relação - coleção de laços de um tipo específico entre membros de um grupo;

v rede social - conjunto finito de atores e as relações entre eles.

Wasserman e Faust (1994) definem que os métodos relacionados à análise de redes podem ser agrupados em propriedades estruturais, como as medidas de centralidade, densidade, transitividade e coesão; papéis e posições, como a análise de equivalência estrutural, regular e local, análise de clusters e de blockmodels; e análise estatística dos relacionamentos, usadas para testar proposições teóricas acerca das propriedades relacionais.

\section{Propriedades estruturais}

A abordagem relacional enfoca as conexões diretas e indiretas entre os atores (Emirbayer e Goodwin, 1994), buscando entender comportamentos e processos por meio da conectividade entre eles. Para tanto, são utilizados principalmente dois grupos de medidas: centralidade e prestígio; e coesão social.

\section{Centralidade}

Um ator é localmente central, se ele apresenta grande número de conexões com outros pontos; será globalmente central, se possuir posição significativamente estratégica na rede como um todo (Scott, 2000). Para tanto, três medidas são mais comumente utilizadas para avaliar a centralidade dos atores em uma rede, que são (Hanneman, 2001; Hanneman e Riddle, 2005; Scott, 
2000; Wasserman e Faust, 1994): centralidade de grau (degree); centralidade de proximidade (closeness); e centralidade de intermediação (betweenness).

A centralidade de grau é medida pelo número de laços que um ator possui com outros atores em uma rede (Wasserman e Faust, 1994). Como a centralidade de grau leva em conta somente os relacionamentos adjacentes, para Scott (2000) tal medida revela somente a centralidade local dos atores.

A centralidade de proximidade é baseada na proximidade ou distância de um ator em relação aos outros atores em uma rede. A medida de centralidade de proximidade (closeness) de um ator é obtida por meio da soma das distâncias geodésicas entre todos os outros atores (Hanneman, 2001; Hanneman e Riddle, 2005; Scott, 2000; Wasserman e Faust, 1994). Segundo Scott (2000), a medida de centralidade de proximidade é indicada para conhecer a centralidade global dos atores.

Na centralidade de intermediação, a interação dos atores não adjacentes pode depender de outros atores, que podem potencialmente ter algum controle sobre as interações dos dois atores não adjacentes. De acordo com Freeman (1979) e Wasserman e Faust (1994), um ator é um intermediário que se liga a vários outros atores que não se conectam diretamente.

\section{Coesão social}

Um dos maiores interesses sociais em análise de rede é identificar subgrupos coesos de atores em uma rede. Em termos estruturais, subgrupos coesos são subconjuntos de atores que apresentam laços relativamente fortes, diretos, coesos, intensos e freqüentes (Wasserman e Faust, 1994). Argumenta-se que subgrupos coesos possuem suas próprias normas, valores, orientações e subculturas (Scott, 2000), sendo base para a solidariedade, identidade e comportamento coletivo em maior intensidade entre esses atores de dentro do grupo do que com os de fora. Esse fenômeno é conhecido como homofilia (De Nooy, Mrvar e Batagelj, 2005).

A noção de subgrupo é formalizada pela propriedade geral de coesão entre membros dos subgrupos, com base em propriedades específicas de seus laços. Desde que tais propriedades possam ser quantificadas, subgrupos coesos podem ser formalizados, expondo as diferentes propriedades de laços (Wasserman e Faust, 1994). Assim, há diferentes modelos teóricos, muitas vezes descritos como cliques, clusters, componentes, cores e ciclos (Scott, 2000). No entanto Wasserman e Faust (1994) afirmam que as idéias conceitualizadas em subgrupos apresentam quatro propriedades gerais que influenciam a for- 
malização desse conceito: mutualidade dos laços; proximidade e alcance entre membros dos subgrupos; freqüência dos laços entre membros; e freqüência relativa de laços entre membros fora e dentro dos subgrupos.

As medidas de subgrupos baseados na mutualidade atêm-se em verificar até que ponto todas as escolhas de pessoas em um subgrupo são mútuas, formando "cliques" entre os atores. Clique é conceito fundamental para estudar a coesão de subgrupos, na qual a teoria dos grafos oferece um conceito formal e preciso. Clique é uma subrede ou subgrafo completo de três ou mais nós, nos quais todos os pontos estão diretamente conectados (De Nooy, Mrvar e Batagelj, 2005; Scott, 2000; Wasserman e Faust, 1994).

A alcançabilidade é uma idéia de subgrupo alternativa que leva em consideração os intermediários do processo social. Para tanto, subgrupos coesos, baseados na alcançabilidade, requerem que a distância geodésica entre os pontos seja pequena, formalizados no conceito de $n$-clique. Um $n$-clique é um subgrupo em que a maior distância geodésica entre dois nós é menor ou igual a $n$ (Wasserman e Faust, 1994), onde $n$ é o caminho máximo em que membros de um clique podem estar conectados (Scott, 2000).

A abordagem $k$-plexes é denominada como subgrafo em que cada nó é adjacente a todos os nós, exceto a $k$ outros pontos, ou seja, subgrafo com $g$ nós em que nenhum dos nós pode conter menos do que $g-k$ laços (Hanneman, 2001; Scott, 2000; Wasserman e Faust, 1994).

A quarta idéia de subgrupos coesos compara a coesão relativa entre membros fora e dentro dos subgrupos. A primeira medida usada para realizar tais comparações é intitulada de LS Sets. Seidman (1983) define um conjunto de nós em uma rede como LS Set, se cada um desses nós apresenta mais laços entre participantes desse conjunto do que com participantes de fora. A segunda medida, Lambda Sets, é uma extensão da noção de LS Sets, desenvolvida por Borgatti, Everett e Shirey (1990). Segundo Wasserman e Faust (1994), um conjunto de nós é um Lambda Set, se algum desses nós apresenta conectividade de linha com algum nó fora do Lambda Set.

\section{Papéis e posições}

Muitos métodos de análise de redes empregam diferentes abordagens para conceitualizar a estrutura social. Muitas dessas estão focadas na análise posicional das relações, atendo-se à natureza ou aos atributos dos atores, tomando senso de certos comportamentos e processos relacionados com os padrões de relações que definem a posição dos atores como relativa a todos os outros 
atores em um sistema social (Emirbayer e Goodwin, 1994). De acordo com Wasserman e Faust (1994), na análise de redes sociais, posição se refere à coleção de indivíduos que são similarmente imersos em uma rede de relações, no tempo em que papel se refere às características das relações obtidas entre atores e posições.

Existem dois aspectos-chave na análise de papéis e posições: identificar as posições sociais como coleções de atores que são similares em seus laços; e modelar papéis sociais como sistemas de laços entre atores ou entre posições. O primeiro aspecto focaliza a avaliação de posições a partir de grupos de atores, utilizando abordagem de equivalência estrutural (Lorrain e White, 1971). O segundo, foca a avaliação de papéis a partir de grupos de relações, contando com métodos de blockmodels (White, Boorman e Breiger, 1976) e de álgebra relacional (Boorman e White, 1976).

\section{Equivalência estrutural}

Dois atores são estruturalmente equivalentes se têm idênticos laços com outros atores em uma rede (Scott, 2000; Wasserman e Faust, 1994). A equivalência estrutural é utilizada na realização de análise de posições; contudo, para Wasserman e Faust (1994), uma completa análise posicional requer quatro passos: definição formal de equivalência; medida de equivalência estrutural; representação das equivalências; e avaliação da adequação da representação.

Para efetuar a medida de posição é necessário especificar uma definição formal, para considerar o grau de equivalência entre os atores, utilizando-se, para tanto, da equivalência estrutural. Em seguida, deve ser estabelecida a medida de equivalência que medirá se um ator é ou não equivalente. Burt (1976) estabelece que a medida de equivalência baseada na distância euclidiana avalia se atores $i$ e $j$ apresentam as mesmas entradas nas linhas e colunas das matrizes (Hanneman, 2001; Hanneman e Riddle, 2005; Scott, 2000; Wasserman e Faust, 1994).

A representação das posições na rede determina os atores em suas classes equivalentes e os relacionamentos entre as classes. O tipo mais comum de representação de uma classe é o modelo discreto, que provê a divisão de atores na rede de acordo com as classes equivalentes. Finalizando, na análise posicional é avaliada a adequação de uma representação. A avaliação de adequação normalmente requer o uso de modelos probabilísticos. Devido à complexidade, essa fase muitas vezes não é realizada. 


\section{Blockmodels}

Para White, Boorman e Breiger (1976), blockmodel é um modelo que apresenta características gerais de uma rede, tanto os laços entre posições, quanto as informações sobre atores individuais. Baseia-se em duas premissas: uma partição de atores em uma rede é chamada de posição; e para cada par de posições é estabelecida a presença ou abstenção de um laço entre as posições (Wasserman e Faust, 1994). Wasserman e Faust (1994) afirmam que há três formas de interpretar um blockmodel: pela validação do blockmodel feita a partir dos atributos dos atores; a partir da descrição das posições individuais; e a partir da descrição do blockmodel como um todo.

Quando há diferença significativa entre posições em relação às características dos membros, há validação externa do blockmodel. Dependendo da orientação teórica, pode-se argüir que as características dos atores são importante determinante de suas redes de relações, observadas na estrutura posicional, ou, por outro lado, a posição estrutural em uma rede influencia o estabelecimento de características entre atores.

Avaliando-se o grau de entrada e de saída entre os blocos, pode-se verificar se as posições apresentam algum tipo de comportamento como, por exemplo, se nós em determinada posição transmitem mais relações do que recebem e vice-versa, ou se a posição está isolada. Entender a estrutura de relacionamento entre as posições contribui para o entendimento do comportamento de atores que estão em uma mesma posição social. A terceira forma de avaliar um blockmodel é considerar a configuração inteira dos laços entre posições, expressa pela matriz imagem.

\section{Análise estatística dos relacionamentos}

De acordo com Wasserman e Faust (1994), existem duas razões para a utilização de métodos estatísticos em análise de redes. A primeira, no caso de grandes redes, é a possibilidade de descrever e entender padrões de comportamento tanto da rede como um todo, quanto de seus atores imersos. A segunda razão é a possibilidade de entender o processo de evolução de redes no tempo, a partir da probabilidade de ações dos atores.

Wasserman e Faust (1994) afirmam que ferramentas estatísticas de análise de redes são usadas principalmente para três finalidades: comparar duas relações no mesmo conjunto de atores; explicar o impacto de atribu- 
tos nos relacionamentos, e vice-versa; e explicar as relações entre os atores na rede.

Historicamente, métodos estatísticos em análise de redes sociais cingiram-se na avaliação de redes em nível local. Assim, dois níveis de análise são utilizados: díades e tríades. O nível de díade se atém basicamente na avaliação de probabilidades de relacionamento entre pares de atores. O outro nível, tríades, foca a análise entre três atores.

A partir do referencial teórico apresentado sobre a análise de redes sociais, que servirá como apoio à interpretação dos dados no presente estudo, serão discutidos os procedimentos metodológicos referentes à coleta, tabulação e análise dos dados.

\section{Procedimentos metodológicos}

Este artigo é descritivo e explicativo: descritivo por apresentar as propriedades estruturais e posicionais das instituições no campo de pesquisa em administração pública e gestão social; explicativo quando verifica como a estrutura de relacionamento afeta a produção científica das instituições. A estratégia de coleta dos dados foi a pesquisa documental, na qual foram avaliados os artigos publicados nos anais do Enanpad (549 artigos), no período de 2000 a 2005.

O método de pesquisa empregado foi quantitativo, mais especificamente a análise de redes, que possibilita avaliar tanto os aspectos descritivos dos relacionamentos, quanto as análises estatísticas causais de tais fenômenos (Hanneman, 2001; Scott, 2000; Wasserman e Faust, 1994). Escolheu-se utilizar o método de análise de redes sociais por incluir informações sobre o relacionamento entre as unidades.

\section{Tabulação dos dados}

Os dados foram tabulados com o uso do software Microsoft Excel ${ }^{\circledR}$. Para efeitos de tabulação dos dados, duas instituições são consideradas conectadas, se algum dos seus pesquisadores produziu artigo conjuntamente, ou seja, são co-autores de uma publicação. Por exemplo, três artigos: artigo A, artigo B e artigo C apresentam a configuração de co-autoria demonstrada no quadro. 


\section{Artigos e suas respectivas co-autorias}

\begin{tabular}{|lc|}
\hline Artigos & \multicolumn{1}{c|}{ Autores } \\
\hline Artigo A & Instituição (1), (2), (6) \\
Artigo B & Instituição (2), (3), (4), (5), (6) \\
Artigo C & Instituição (7), (6) \\
\hline
\end{tabular}

Cada artigo apresenta $n$ número de instituições, cada uma representada por um número. Observe que a instituição (6) colaborou em três artigos, a instituição (2) em dois artigos (artigos A e B) e o restante colaborou somente em um único artigo. A partir de tais informações, foi construída uma rede de relações em formato 2-mode que, depois, foi transformada em rede 1-mode, ou seja, em matriz quadrática.

\section{Análise dos dados}

A análise de redes foi feita por meio dos softwares Ucinet 6.0 e Pajek 1.10. Em relação às propriedades estruturais, foram avaliadas a estrutura da rede e seus componentes, as medidas de centralidade e as medidas de coesão, a partir das medidas de cliques, $n$-cliques e cut-points. Para as medidas posicionais da rede, foram utilizadas as análises de equivalência estrutural e a técnica de blockmodeling, pela qual foi extraída a visão global dos relacionamentos a partir da permutação dos relacionamentos baseados em medidas categóricas no tocante à unidade federativa em que a instituição se localiza.

A avaliação da relação entre os indicadores de centralidade da rede e os indicadores de produção científica foi realizada por meio do software SPSS $13^{\circledR}$, a partir de dados apresentados no trabalho de Hocayen-da-Silva, Rossoni e Ferreira Júnior (2008). Para tanto, foram utilizadas duas variáveis dependentes: a primeira relativa ao número de artigos publicados por instituição; a segunda em relação ao número de autorias por instituição, ou seja, quantas vezes autores de certa instituição colaboraram em algum artigo. Primeiro, criavam-se duas matrizes de correlação (uma para cada variável dependente) com todas as variáveis, sendo verificadas as correlações entre as variáveis de produção científica e de número de autoria com as variáveis de centralidade. Buscou-se também verificar se alguma das variáveis apresentava multicolinearidade. Depois de as variáveis significativas terem sido identificadas, foram construídos os modelos explicativos, a partir de regres- 
são linear. Aceitavam-se como significativos, os resultados que apresentaram valor de $p<0,05$.

\section{Discussão e análise dos resultados}

Na figura 1, pode ser observada a estrutura de relacionamento entre as instituições de pesquisa na área de administração pública e gestão social. Nem todas as instituições estão diretamente conectadas, formando diversos componentes isolados. Na figura, foram excluídas aquelas que não cooperaram com nenhuma outra.

Figura 1

Rede de colaboração entre instituições de pesquisa em administração pública e gestão social

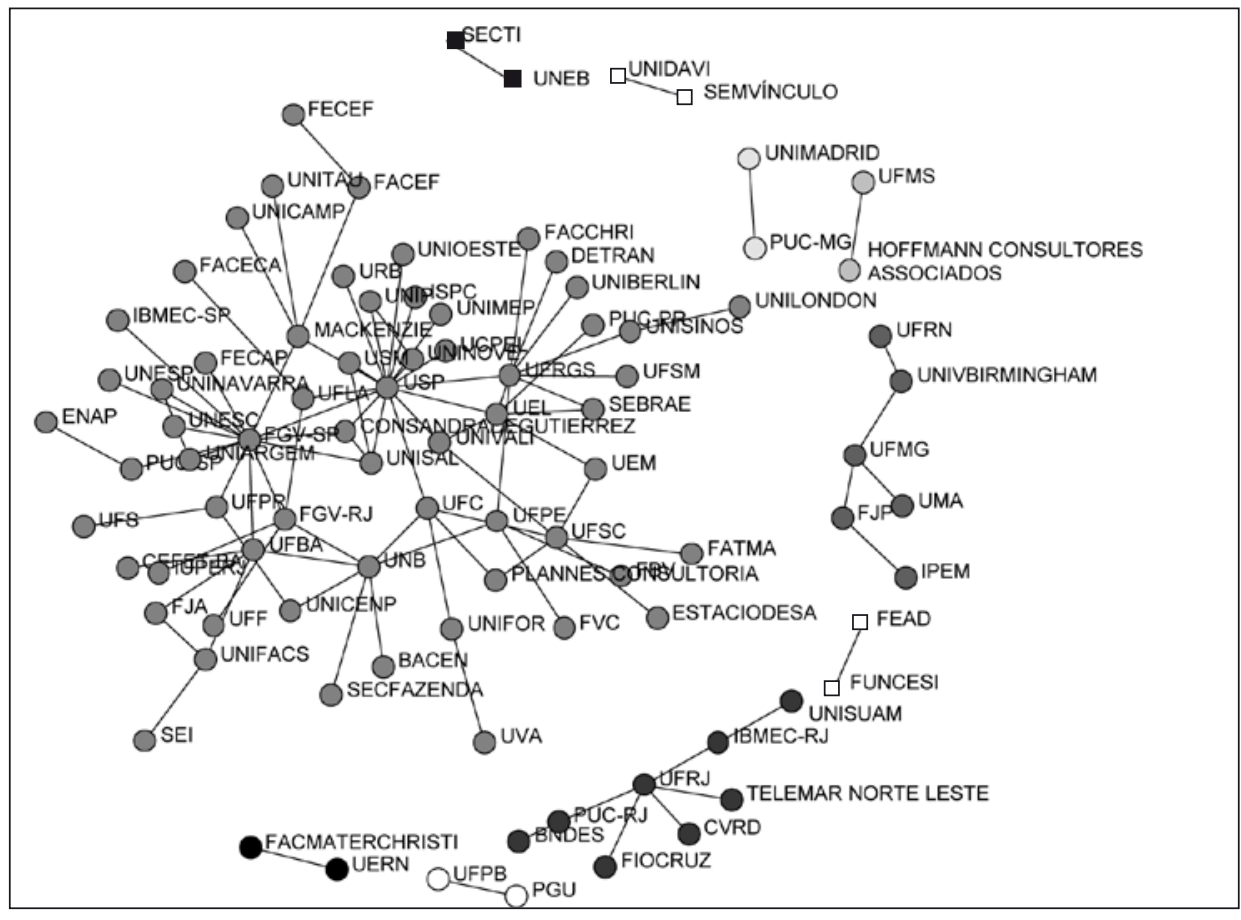

Das 120 instituições de pesquisa, 29 estão isoladas (24\%), ou seja, não cooperaram com nenhuma outra no desenvolvimento da produção científica. Entretanto, apesar do grande percentual de instituições isoladas, 63 (52,5\%) 
estão diretamente ou indiretamente interligadas, formando o componente principal. As 63 instituições que formam tal componente estão na rede à esquerda na figura 1. Tais resultados são semelhantes com os encontrados por Rossoni, Hocayen-da-Silva e Ferreira Júnior (2008) no campo de pesquisa em ciência e tecnologia, no qual verificaram que o componente principal englobava $57,3 \%$ das instituições. Tal estudo também coletou dados a partir de artigos apresentados entre os períodos de 2000 e 2005, o que facilita a comparação dos resultados. Devido à semelhança entre o tamanho percentual dos componentes principais nos dois campos distintos, pode-se acreditar que outros campos da área da administração apresentem mesma configuração estrutural, com aproximadamente metade das instituições interligadas. Ainda assim, estudos em outras áreas da administração devem ser feitos para inferir se há um padrão estrutural nos campos de pesquisa.

Além do componente principal, existem mais nove componentes que fizeram parte do campo de pesquisa em administração pública e gestão social. Porém, como exposto na tabela 1 , o tamanho dos componentes é bem inferior ao tamanho do componente principal, apresentando grupos de colaboração com, no máximo, oito instituições. Diante de tal quadro, pode-se considerar o campo de pesquisa em administração pública e gestão social relativamente fragmentado, com mais de $40 \%$ das instituições isoladas do maior grupo de colaboração. Todavia existe um grande componente que engloba mais da metade das instituições.

Tabela 1

Distribuição de freqüência entre os componentes

\begin{tabular}{|ccccc|}
\hline Componente & Freq & Freq $\%$ & CumFreq & CumFreq $\%$ \\
\hline Isoladas & 29 & 24,1667 & 29 & 24,1667 \\
1 & 63 & 52,5000 & 92 & 76,6667 \\
2 & 8 & 6,6667 & 100 & 83,3333 \\
3 & 2 & 1,6667 & 102 & 85,0000 \\
4 & 2 & 1,6667 & 104 & 86,6667 \\
5 & 6 & 5,0000 & 110 & 91,6667 \\
6 & 2 & 1,6667 & 112 & 93,3333 \\
7 & 2 & 1,6667 & 114 & 95,0000 \\
8 & 2 & 1,6667 & 116 & 96,6667 \\
9 & 2 & 1,6667 & 118 & 98,3333 \\
10 & 2 & 1,6667 & 120 & 100,0000 \\
\hline Sum & & & 120 & 100,0000 \\
\hline
\end{tabular}


Em relação à configuração estrutural entre as instituições de pesquisa, observa-se que, apesar de a densidade da rede ser baixa $(1,4 \%)$, com pequeno grau de centralização (13,10\%), existe grande número de instituições que estão interligadas, nas quais as distâncias médias entre elas são pequenas $(3,32)$; ou seja, apesar de o campo ter baixa densidade, na média, uma instituição para se relacionar com outra precisa basicamente de três intermediários.

Tal fato tem importantes implicações para o campo de pesquisa em administração pública e gestão social. Apesar da baixa densidade geral, o coeficiente de agrupamento entre as instituições não é tão baixo $(0,299)$, possibilitando que tais instituições tenham proximidade suficiente para formação de capital social, a partir da coesão (Coleman, 1990). Além disso, tais organizações apresentam uma distância média relativamente pequena com todas as outras organizações $(3,32)$, possibilitando a troca de informações de forma dinâmica dentro do campo. Essa proximidade entre as instituições abre espaço para a troca de experiências entre as diversas instituições, mantendo canal aberto para que a inovação na produção científica aconteça mais rapidamente, possibilitando outra forma de construção do capital social: a partir dos laços fracos (Granovetter, 1973) e dos buracos estruturais (Burt, 1992). Como já abordado por Kogut e Walker (2001) e Uzzi e Spiro (2005), quando esses dois mecanismos geradores de capital social (coesão de um lado, laços fracos e buracos estruturais de outro) estão presentes, a estrutura de um campo apresenta uma configuração que é propícia para a geração de capital social. Tal configuração é conhecida como small world ou pequenos mundos (Watts e Strogatz, 1998). Em pequenos mundos, os atores estão conectados localmente de forma mais coesa; contudo eles apresentam laços fora desses grupos, interligando globalmente a grande maioria desses atores, o que possibilita a rápida interação desses diversos grupos locais.

Novamente, todos os indicadores estruturais da rede, apresentados acima, têm bastante semelhança com os indicadores estruturais no campo de pesquisa em ciência e tecnologia (Rossoni, Hocayen-da-Silva e Ferreira Júnior, 2008), o que reforça a possibilidade de campos distintos apresentarem padrões estruturais similares.

Em relação aos componentes, o de maior tamanho se destaca também pelo volume de produção, apresentando as instituições que são mais prolíficas como, por exemplo, FGV-SP, FGV-RJ, UFBA, UFRGS, UnB, USP (Hocayen-daSilva, Rossoni e Ferreira Junior, 2008). Fato que é positivo no campo, pois aquelas instituições que realmente produzem o conhecimento na área não são tão endogênicas, mantendo laços de pesquisa com as outras instituições que são também prolíficas. Dessa forma, faz-se necessário avaliar as medidas de 
ego das instituições para elucidar melhor o papel desses agentes no campo de pesquisa em administração pública e gestão social. Para tanto, algumas propriedades estruturais das instituições de pesquisa serão elucidadas, no caso as medidas de centralidade e de coesão da rede.

No tocante à centralidade de grau, a instituição que manteve o maior número de laços com outras instituições no período foi a USP, como evidenciado na tabela 1. De todos os laços possíveis, a instituição manteve laços com mais de $14 \%$ de todas as outras. Entretanto, apesar de a instituição possuir o maior número de laços, ela é a quinta instituição em número de publicações na área, com produção bastante inferior à de instituições como FGV-SP, FGV-RJ e UFBA. Isso mostra que, mesmo tendo produção significativamente inferior a outras instituições, ela tem importante papel como elo entre várias instituições. Além da USP, a segunda instituição que manteve maior número de colaborações diretas foi a FGV-SP, com 14 laços: instituição que apresenta o maior volume de produção na área.

Além de USP e Unicamp, outras instituições apresentam significativo número de laços, como, por exemplo, UFRGS e UnB, com nove e sete laços, respectivamente. As instituições com grande número de laços apresentam papel fundamental na estrutura do campo, pois servem como elos principais entre grande parte das instituições, agregando elevado número de instituições em sua volta.

Vale destacar que duas instituições, com grande volume de produção científica na área (FGV-RJ e UnB), apresentam relativamente poucos laços com outras instituições. Subentende-se que os pesquisadores de tais instituições busquem preferencialmente cooperar entre os pares da própria instituição, o que, teoricamente, pode gerar redundância de informação, sendo obstáculo à heterogeneidade na produção do conhecimento na área.

A importância de uma instituição na rede não se dá somente pelo número de contatos diretos que ela mantém, mas também pelo número de contatos que intermedeia. Instituições que são intermediárias de diversas outras podem controlar o fluxo de informação entre elas, estabelecendo uma relação de dependência com aquelas que necessitam de suas conexões. Além disso, servem como ponte para o desencadeamento da informação, sendo mecanismo gerativo de inovação. Como se esboça na tabela 3 , as três instituições com maior centralidade de intermediação, USP, FGV-SP, UFRGS e UnB, coincidentemente também são as que apresentam maior número de laços (tabela 2). Reforça-se o papel dessas três instituições não só como fonte de ligação com outras instituições, mas também como importantes intermediárias na construção do conhecimento no campo de pesquisa em administração pública e gestão social. 
Tabela 2

As 10 instituições com maior número de laços

\begin{tabular}{|lcccc|}
\hline Instituição & Degree & NrmDegree & Share & Artigos \\
\hline USP & 17.000 & 14.286 & 0,085 & 24 \\
FGV-SP & 14.000 & 11.765 & 0,070 & 75 \\
UFRGS & 9.000 & 7.563 & 0,045 & 28 \\
UnB & 7.000 & 5.882 & 0,035 & 28 \\
UEL & 6.000 & 5.042 & 0,030 & 10 \\
UFSC & 6.000 & 5.042 & 0,030 & 14 \\
FGV-RJ & 5.000 & 4.202 & 0,025 & 74 \\
Mackenzie & 5.000 & 4.202 & 0,025 & 7 \\
UFRJ & 5.000 & 4.202 & 0,025 & 3 \\
UFC & 5.000 & 4.202 & 0,025 & 1 \\
UFBA & 5.000 & 4.202 & 0,025 & 63 \\
\hline
\end{tabular}

Observa-se que a FGV-RJ tem papel limitado na intermediação entre outras instituições, o que pode ocasionar que sua produção científica, apesar de vasta, tenha pouca repercussão no desenvolvimento do conhecimento da área. Ressalta-se que tal avaliação é de caráter meramente estrutural, pois não se discute aqui a qualidade de tais pesquisas, mas as condições estruturais propícias para o seu desenvolvimento.

Tabela 3

As 10 instituições com maior centralidade de intermediação

\begin{tabular}{|lrc|}
\hline Instituição & Betweenness & nBetweenness \\
\hline USP & 1.022 .900 & 14.569 \\
FGV-SP & 754.867 & 10.752 \\
UFRGS & 454.050 & 6.467 \\
UnB & 331.433 & 4.721 \\
UFC & 320.083 & 4.559 \\
UFBA & 264.500 & 3.767 \\
Mackenzie & 237.000 & 3.376 \\
UFPE & 183.433 & 2.613 \\
FGV-RJ & 179.167 & 2.552 \\
UEL & 145.117 & 2.067 \\
\hline
\end{tabular}


Além do número de laços que as instituições mantêm e suas capacidades de intermediação, pode-se analisar quanto um ator está centralizado globalmente em uma rede, isto é, qual instituição, na média, está mais próxima do conjunto de instituições que fazem parte da rede. Como nas duas outras medidas de centralidade anteriores, pode-se ver que a instituição mais globalmente centralizada é a USP, pois apresenta a menor distância média entre todas as instituições da rede (tabela 3). A FGV-SP também se apresenta globalmente centralizada, estando próxima de muitas outras instituições de pesquisa. Instituições como UFRGS e UnB se mostram menos globalmente centralizadas do que localmente. Nas medidas de centralidade de intermediação e de grau, as duas se mostraram entre as quatro mais centrais. Contudo seus escores de centralidade de proximidade foram inferiores aos das medidas anteriores, o que indica que tais instituições apresentam mais influência local do que global. Destaca-se novamente a posição da UFBA e da FGV-RJ que novamente apresentaram baixo índice de centralidade global, com a segunda instituição não se enquadrando nem entre as 10 mais centrais por proximidade.

Tabela 4

\section{As 10 instituições com maior centralidade de proximidade*}

\begin{tabular}{|lcc|}
\hline Instituição & Farness & nCloseness \\
\hline USP & 126.000 & 49.206 \\
FGV-SP & 139.000 & 44.604 \\
UFC & 155.000 & 40.000 \\
Mackenzie & 159.000 & 38.994 \\
UFRGS & 160.000 & 38.750 \\
UnB & 165.000 & 37.576 \\
Unisal & 165.000 & 37.576 \\
Agutierrez & 166.000 & 37.349 \\
UEL & 167.000 & 37.126 \\
UFBA & 172.000 & 36.047 \\
\hline
\end{tabular}

* Levaram-se em conta somente os nós do componente principal.

As 10 instituições mais globalmente centrais também estão bem próximas umas das outras (ver círculo central da figura 2), apresentando pequena distância entre elas, criando um núcleo pelo qual a informação circula di- 
namicamente, o que possibilita que o conhecimento seja compartilhado em primeira mão entre essas instituições.

Entretanto, apesar de estarem visualmente próximas, isso não significa que elas fazem parte de um grupo coeso.

\section{Figura 2}

Componente principal da rede entre instituições de pesquisa em administração pública

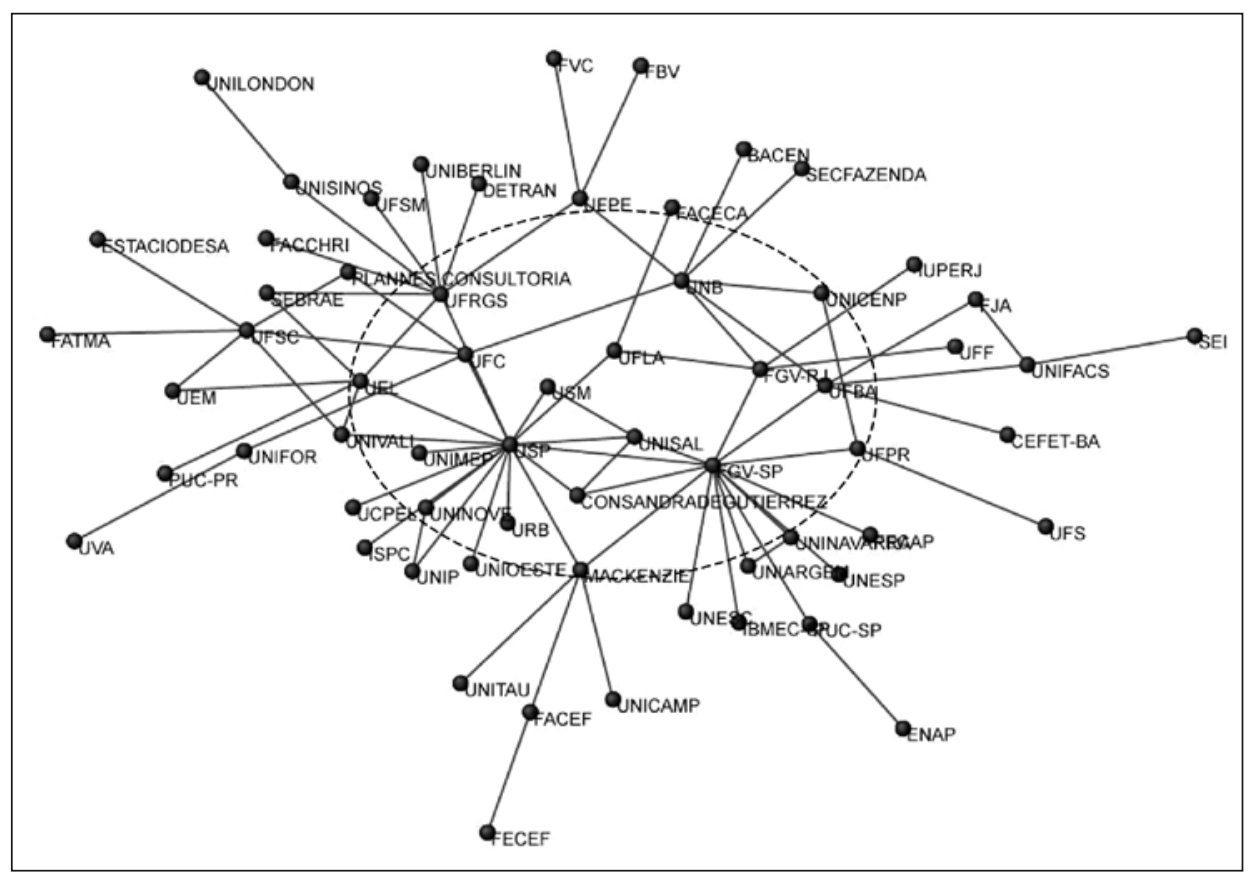

As 120 instituições que formaram o campo de produção científica em administração pública e gestão social apresentam 10 cliques de tamanho igual a três. Entre esses cliques, a USP participa de seis, número bem superior ao apresentado pelas demais instituições. Nesses termos, a instituição atua como elo desses diferentes grupos, conectando-os. Instituições que participam de diferentes grupos têm papel importante na rede, pois tanto integram tais grupos em um grupo maior, como servem de mecanismos isomórficos de estabelecimento de padrões de produção científica. No campo de ciência e tecnologia, apesar de ter quase o dobro de instituições que produziram pesquisa no período, o número de cliques foi quatro vezes maior, com 40 cliques de três institui- 
ções (Rossoni, Hocayen-da-Silva e Ferreira Júnior, 2008), o que indica que na área de administração pública e gestão social há menor proporção de cliques.

Porém, se for avaliado o número de clique de tamanho igual a quatro, existe somente um clique formado pelas instituições FGV-SP, Unisal e USP e pelo Consórcio Andrade Gutierrez. Pode-se entender que, no campo de pesquisa em administração pública e gestão social, os grupos de pesquisa apresentam pequeno número de instituições envolvidas, muitas dessas atuando localmente, ou com parceiros em estados vizinhos. Isso leva a crer que a colaboração entre as instituições não é prática rotineira, tendo muitas vezes somente o objetivo de realizar, no máximo, uma pesquisa isoladamente. Tais pesquisas provavelmente não têm continuidade no decorrer do tempo, nem apresentam envolvimento de novas instituições, o que dificulta a construção do conhecimento na área de forma acumulativa.

Se for avaliada a coesão da rede em relação à alcançabilidade e ao diâmetro dos grupos, pode-se verificar que existem 38 2-cliques, nos quais a distância máxima entre todas as instituições é de dois passos. Desses, há dois grandes grupos: o primeiro apresenta 16 instituições, tendo como mais representativas, USP, FGV-SP e UFRGS; o segundo apresenta 15 instituições, com FGV-RJ e UFBA como representativas, mas também com a USP e a FGV-SP, entre elas. Pode-se verificar que tanto a FGV-SP, quanto a USP são importantes elos desses grupos, atuando como meio de troca do conhecimento desenvolvido entre grupos distintos.

Além da avaliação da coesão a partir dos grupos, há 24 instituições que são consideradas cut-points, ou seja, se forem removidas aumentarão o número de componentes na rede, fragmentando-a. Se for observado que a rede apresenta 120 instituições, com 63 delas no componente principal, há grande proporção de instituições que mantêm a rede integrada. Todavia, se essas deixarem de desenvolver pesquisas com outras instituições, podem ocasionar maior fragmentação do campo.

\section{Análise posicional das relações no campo de pesquisa em administração pública}

Outra tradição em análise de redes sociais vai além dos elementos estruturais centralidade e coesão: é a análise posicional das relações. Apesar de as duas análises terem elucidado a configuração do campo de pesquisa em administração pública e gestão social no Brasil, as instituições podem apresentar certos padrões de interação que estão intimamente relacionados com a posição que 
essas ocupam no campo de pesquisa. Para avaliar a posição que as instituições ocupam no campo de pesquisa em administração pública e gestão social, foi realizada a avaliação da equivalência estrutural.

A partir do algoritmo Concor, verificou-se que existem sete grupos de instituições estruturalmente equivalentes no campo. O agrupamento das instituições, a partir da equivalência estrutural, é feito de acordo com a similaridade entre os laços que todas as instituições mantêm, criando grupos de acordo com a correlação entre os laços diretos.

Dos sete grupos, três são formados por instituições que não fazem parte do componente principal: um deles, em que constam somente instituições isoladas; outro formado predominantemente por instituições que fazem parte do componente com instituições fluminenses por instituições que apresentam somente um laço de colaboração; o outro é formado exclusivamente pelo componente das instituições mineiras, com exceção da UFRN e da Universidade de Birmingham (ver figura 1). Os três grupos não apresentaram nenhum tipo de colaboração entre si, nem com os outros quatro grupos restantes, o que expõe o isolamento de tais grupos de instituições do componente principal. Estruturalmente, tais grupos de instituições estão em posição periférica no campo, não compartilhando laços de pesquisa com as instituições mais centrais, tanto em termos estruturais, quanto em termos de volume de produção.

Os quatro grupos restantes são representados por instituições que formam o componente principal. Mesmo estando posicionadas em grupos distintos, apresentam algum canal de relacionamento. Um desses grupos apresenta 13 instituições que são estruturalmente equivalentes à FGV-RJ. Já outro, também com 13 instituições, tem a USP como instituição de relevância no campo. Os outros dois grupos apresentam duas instituições de grande produção cada: um deles formado por 14 instituições encabeçadas pela FGV-SP e a UFRGS; o outro composto por 22 instituições, que estão organizadas em volta da UFBA e da UnB.

Instituições que são estruturalmente equivalentes tendem a apresentar papéis semelhantes no campo de pesquisa, levando a informação a ser redundante por meio da equivalência estrutural, e não pela coesão (Burt, 1992), o que possibilita que elas estabeleçam mecanismos de homofilia, a partir da similaridade de seus papéis.

Os papéis de tais instituições no campo podem ser avaliados a partir do seu posicionamento em termos regionais. Assim, buscou-se avaliar como as relações se dão entre os pesquisadores, no tocante ao estado em que estão localizadas as instituições. Para efeitos de análise, foram computados todos os relacionamentos individuais entre pesquisadores, que foram vistos de for- 
ma global, identificando o padrão de relação entre as unidades da federação. Como pode ser visualizado na figura 3 , os vínculos com pesquisadores filiados às instituições do estado de São Paulo estão presentes entre oito estados diferentes, apesar de o Paraná e o Distrito Federal também apresentarem vários vínculos com instituições de outros estados (seis estados). Instituições do Rio Grande do Sul e do Ceará também apresentam laços com instituições de cinco diferentes estados. Também há o contrário: instituições que só mantêm laços com instituições dentro do seu próprio estado como Tocantins, Goiás, Espírito Santo e Mato Grosso.

Figura 3

Visão global das relações entre instituições de diferentes unidades federativas

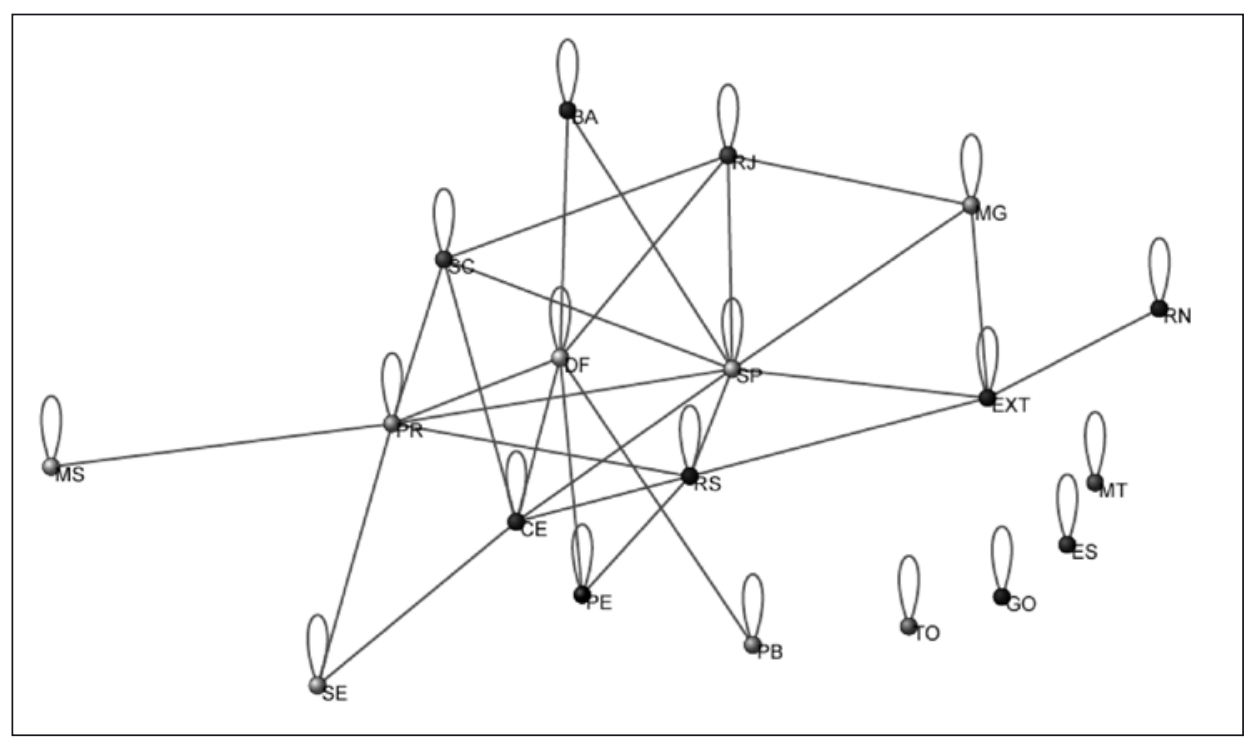

Pode-se verificar que pesquisadores de estados com produção mais periférica buscam apoio em pesquisadores de instituições de maior porte para desenvolver suas pesquisas, o que leva as instituições centrais a terem papel fundamental na construção do conhecimento no campo da administração pública e gestão social. Entretanto, se avaliada a proporção de laços que os pesquisadores mantêm com pesquisadores fora do estado, em relação ao total de relacionamentos que os pesquisadores localizados nesses estados mantiveram, conclui-se que a colaboração entre pesquisadores da área ocorre primordialmente dentro dos limites da unidade federativa. 
Tabela 5

Centralidade e número de laços das unidades federativas

\begin{tabular}{|lcccc|}
\hline UF & Grau & Laços rec. & Laços & \% laços fora \\
\hline SP & 8 & 615 & 32 & 5,1 \\
PR & 6 & 221 & 32 & 14,1 \\
DF & 6 & 157 & 12 & 7,4 \\
CE & 5 & 92 & 14 & 14,4 \\
RS & 5 & 168 & 19 & 11,0 \\
EXT & 4 & 21 & 8 & 32,0 \\
RJ & 4 & 276 & 9 & 3,2 \\
SC & 4 & 125 & 20 & 15,5 \\
MG & 3 & 247 & 5 & 2,0 \\
SE & 2 & 10 & 2 & 16,7 \\
PE & 2 & 77 & 2 & 2,5 \\
BA & 2 & 389 & 2 & 0,5 \\
MS & 1 & 1 & 1 & 50,0 \\
PB & 1 & 1 & 1 & 50,0 \\
RN & 1 & 39 & 1 & 2,5 \\
MT & 0 & 1 & 0 & 0,0 \\
GO & 0 & 1 & 0 & 0,0 \\
TO & 0 & 1 & 0 & 0,0 \\
ES & 0 & 2 & 0 & 0,0 \\
\hline
\end{tabular}

De acordo com a tabela 5, das 18 unidades federativas identificadas no estudo, 11 delas apresentam mais de $90 \%$ de seus laços entre pesquisadores da própria unidade federativa e 16 delas mais de $80 \%$ dos laços, o que leva a crer que, apesar do crescente avanço da tecnologia de informação, a produção científica na área ocorre primordialmente em nível local. Estados como SP, RJ, BA, MG e o Distrito Federal, que apresentam elevada produção científica na área, são alguns dos estados que contam com maior número de laços recíprocos entre os autores. Como extremo, as instituições baianas só mantiveram laços de cooperação com dois autores de outros estados.

Se forem observados estados com produção intermediária (RS, PR, SC e $\mathrm{CE}$ ), ou mais periférica na área como SE, MS, MT e PB, eles apresentam, percentualmente, maior número de laços com pesquisadores de fora do que os estados com maior produção científica. Há exceções, como PE e RN, que apresentaram pouquíssimos laços e alguns estados isolados; mas, mesmo assim, apresenta-se 
uma tendência de que pesquisadores, localizados em estados que não apresentam produção representativa, busquem relacionar-se mais freqüentemente com pesquisadores de instituições localizadas em estados mais centrais.

\section{Os indicadores de redes têm relação com a produção científica?}

Diante dos elementos estruturais e posicionais da rede entre instituições do campo de pesquisa em administração pública e gestão social, buscou-se compreender, por meio da análise de regressão, como os indicadores de centralidade das instituições causam impacto na sua produção científica. Para tanto, duas variáveis independentes foram selecionadas: uma com o número de artigos publicados por instituição (tabela 6); outra com o número de autorias por instituição, isto é, quantas vezes autores de uma dada instituição colaboraram na produção de um artigo (tabela 7).

A partir da análise de correlação dos indicadores de centralidade com os indicadores de produção, verificou-se que a centralidade de grau e a centralidade de intermediação apresentam correlação positiva e significativa, tanto com a produção quanto com a autoria $(p<0,01)$. Em relação à produção científica, a correlação foi 0,670 para grau e 0,656 para a intermediação. Diante da correlação positiva e significante de tais indicadores, foram realizados três modelos de regressão, expostos na tabela 6 .

Tabela 6

Impacto da centralidade na produção científica

\begin{tabular}{|lccc|}
\hline & Modelo 1 & Modelo 2 & Modelo 3 \\
\hline Grau & $3,283^{*}$ & & $2,649^{*}$ \\
nBetweenness & & $0,05^{*}$ & 0,001 \\
R & 0,738 & 0,695 & 0,741 \\
R2 & $54,4 \%$ & $48,4 \%$ & $54,9 \%$ \\
Sig. & $<0,001$ & $<0,001$ & $<0,001$ \\
\hline$* \mathrm{p} \leq 0,001$ & $\mathrm{n}=91$ & & \\
\hline
\end{tabular}

Já em relação ao número de autorias, a correlação foi 0,742 para grau e 0,738 para a intermediação. Com tais correlações positivas e significantes, foram realizados ainda três modelos de regressão, expostos na tabela 7 .

Nos dois casos, os modelos de número 1 apresentam a relação entre centralidade de grau e produção científica. Segundo o modelo, o grau corresponde a $54,4 \%$ da explicação da produção científica e a $61 \%$ do número de autorias, o que indica que, quanto mais central uma instituição é, mais 
produção ela terá e maior será o número de autorias. Já os modelos de número 2 avaliam a relação entre centralidade de intermediação e a produção científica, explicando $48,4 \%$ da variância relativa à produção e $58 \%$ relativa ao número de autorias. Contudo, a partir da regressão múltipla (modelo 3), a centralidade de intermediação não se mostra significativa, quando usada em conjunto com a centralidade de grau no tocante à produção, mas ainda se mantém significativa em relação ao número de autorias. Em ambos os casos, a centralidade de grau tem maior poder explicativo, indicando que a quantidade produzida de pesquisa está mais ligada aos laços diretos e locais que uma instituição mantém do que com a sua capacidade de intermediação.

Tabela 7

Impacto da centralidade no número de autorias

\begin{tabular}{|lrcc|}
\hline & Modelo 1 & Modelo 2 & Modelo 3 \\
\hline Grau & $6,193^{*}$ & & $3,883^{*}$ \\
nBetweenness & & $0,009^{*}$ & $0,004^{* *}$ \\
R & 0,781 & 0,761 & 0,798 \\
R2 & $61,0 \%$ & $58,0 \%$ & $63,6 \%$ \\
Sig. & $<0,001$ & $<0,001$ & $<0,001$ \\
\hline$* \mathrm{P} \leq 0,001$ & $* * \mathrm{P}<0,01$ & $\mathrm{n}=120$ & \\
\hline
\end{tabular}

Para melhor elucidar tal relação, na figura 4 pode-se observar a relação entre o número de laços e o número de autorias. As instituições (representadas pelos nós) estão dispostas mais ao alto, de acordo com o número de laços que apresentam. Os números de laços de cada instituição estão indicados dentro dos nós. Já o número de autorias está indicado pelo tamanho dos nós: quanto maior o nó, maior o número de autorias.

É visível que os nós de maior tamanho se apresentam predominantemente mais elevados na figura, com os nós de menor tamanho concentrados primordialmente na base. Dessa forma, pode-se visualizar caso a caso o que já tinha sido demonstrado estatisticamente pela análise de regressão. Algumas instituições com menor produção se encontram mais elevadas na figura, indicando que, apesar do razoável número de laços, não produziram tanto no período, o que levou o modelo 1 da regressão a apresentar poder explicativo inferior ao do modelo desenvolvido no campo de ciência e tecnologia, que 
apresentou poder explicativo de 86,9\% (Rossoni, Hocayen-da-Silva e Ferreira Júnior, 2008). Portanto, na área de administração pública e gestão social, a relação entre produção científica e centralidade de grau mostrou-se mais fraca do que na área de ciência e tecnologia.

Figura 4

Rede entre instituições de pesquisa ajustadas para representar

o grau e a autoria

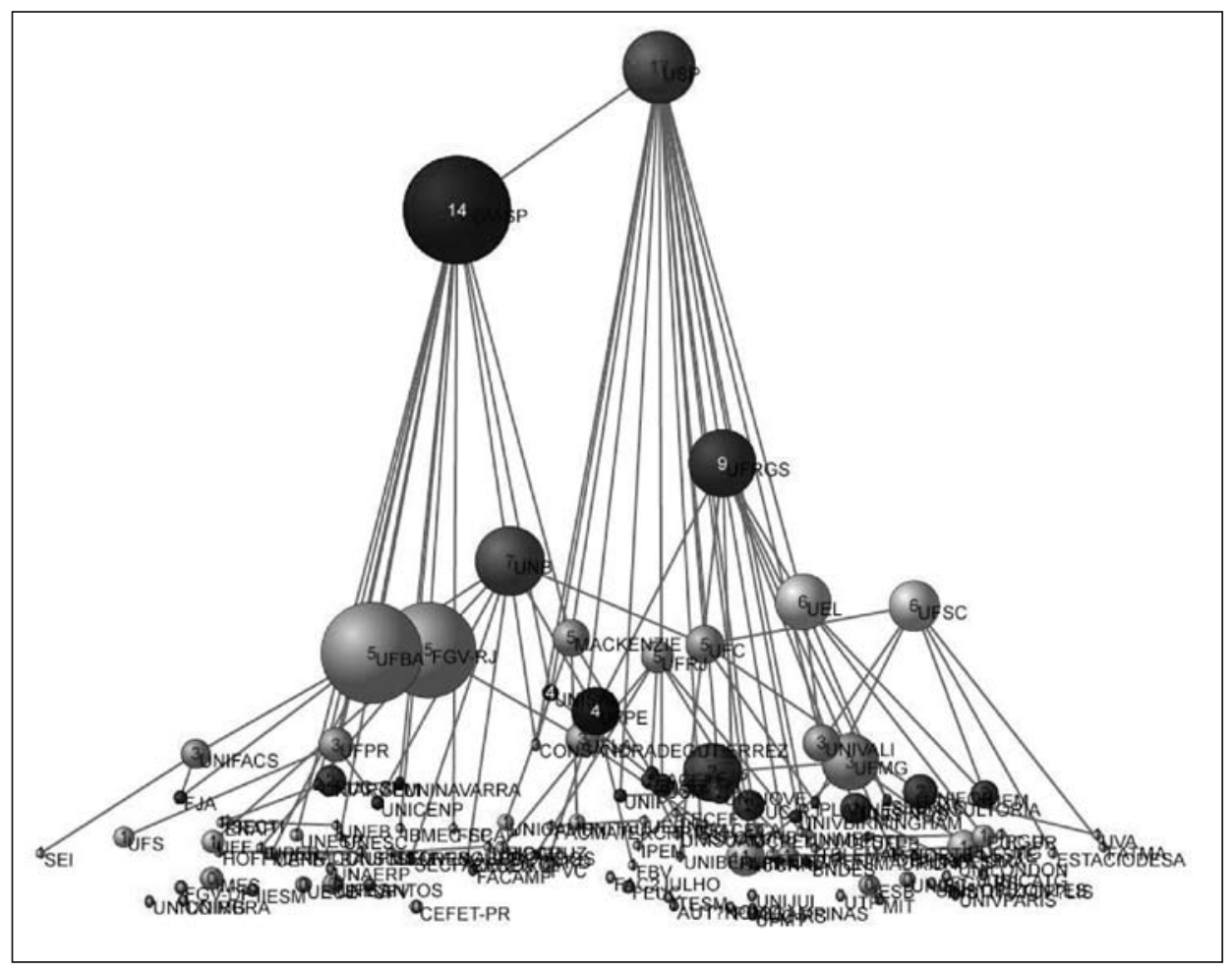

É necessário ressaltar que não se afirma neste artigo que a centralidade causa maior produção ou maior número de autorias, mas que os dois indicadores mantêm uma relação de dualidade que, em conjunto, contribuem tanto para a maior construção de laços, quanto para o aumento do número de artigos produzidos por uma instituição. Nesses termos, pode-se afirmar que a posição que uma instituição ocupa na rede de pesquisa do campo de administração pública e gestão social apresenta relação direta com sua produção científica. 


\section{Considerações finais}

Este artigo apresentou as propriedades estruturais e posicionais da rede de relações entre as instituições do campo de administração pública e gestão social, bem como verificou se tais propriedades apresentam relação com os indicadores de produção científica da área.

Com a análise da rede, pode-se verificar que das 120 instituições que produziram conhecimento científico na área, sob a forma de artigos, 63 estão conectadas, formando um componente principal. Apesar da baixa densidade, a distância entre as instituições é pequena, bastando somente três intermediários para qualquer contato dentro da rede. Além disso, pode-se observar que a USP, FGV-SP e UFRGS são as instituições que apresentam maior centralidade na rede. Pode-se verificar também que os grupos totalmente coesos são pequenos, compostos, na maioria, por somente três instituições. Entretanto, se forem avaliados grupos com duas distâncias geodésicas no máximo, verifica-se que existem dois grandes grupos principais: um liderado pela USP, FGV-SP e UFRGS, o outro liderado pela FGV-SP e pela UFBA.

No que concerne aos elementos posicionais da rede, verificou-se que a estrutura das relações é fortemente conformada por questões regionais, apresentando a maioria dos laços entre os pesquisadores dentro dos limites estaduais. Porém existem instituições com papéis bem distintos, algumas com papel mais central.

A partir da análise de regressão linear, foi encontrada relação forte e significativa entre a centralidade de grau e de intermediação com os indicadores de produção científica e com o número de autorias, tendo a primeira centralidade maior poder explicativo diante das duas variáveis; contudo não se pode afirmar que exista relação de causalidade entre estrutura da rede e produção científica, mas uma relação de dualidade.

Portanto, diante de todas as análises elucidadas neste artigo, afirma-se que a construção do conhecimento científico na área de administração pública e gestão social é fortemente influenciada pela estrutura de relacionamento entre pesquisadores e instituições.

\section{Referências bibliográficas}

BARABASI, A. et al. Evolution of the social network of scientific collaborations. Physica A, v. 311, p. 590-614, 2002. 
BOORMAN, S. A.; WHITE, H. C. Social structure from multiple networks II. Role structures. American Journal of Sociology, v. 81, p. 1384-1446, 1976.

BORGATTI, S. P.; EVERETT, M. G.; SHIREY, P. R. LS sets, lambda sets, and other cohesive subsets. Social Networks, v. 12, p. 337-358, 1990.

BURT, R. S. Structural holes: the social structure of competition. Cambridge: Harvard University Press, 1992.

; LIN, N. Network time series from archival records. In: HEISE, D. R. (Ed.). Sociological methodology. San Francisco: Jossey-Bass, 1976.

COLEMAN, J. Foundations of social theory. Chicago: University of Chicago Press, 1990.

DE NOOY, W.; MRVAR, A.; BATAGELJ, V. Exploratory social network analysis with Pajek. New York: Cambridge University Press, 2005.

EMIRBAYER, M.; GOODWIN, J. Network analysis, culture and the problem of agency. American Journal of Sociology, v. 99, n. 6, p. 1411-1454, May 1994.

FREEMAN, L. C. Centrality in social networks I. Conceptual clarification. Social Networks, v. 1, p. 215-239, 1979.

. Turning a profit from mathematics: the case of social networks. Journal of Mathematical Sociology, v. 10, p. 343-360, 1984.

GRANOVETTER, M. S. The strength of weak ties. American Journal of Sociology, v. 78, n. 6, p. 1361-1380, 1973.

GUIMERA, R. et al. Team assembly mechanisms determine collaboration network structure and team performance. Science, v. 308, p. 697-702, Apr. 2005.

HANNEMAN, R. A. Introduction to social network methods. Riverside: University of California, 2001.

; RIDDLE, M. Introduction to social network methods. Riverside: University of Califórnia, 2005. Disponível em: < http://faculty.ucr.edu/ hanneman/nettext/ index.html>. Acesso em: 2 maio 2006.

HOCAYEN-DA-SILVA, A. J.; ROSSONI, L.; FERREIRA JÚNIOR, I. Administração pública e gestão social: a produção científica brasileira entre 2000 e 2005 . Revista de Administração Pública, v. 42, n. 4, p. 655-680, jul./ago. 2008.

KOGUT, B.; WALKER, G. The small world of Germany and the durability of national networks. American Sociological Review, v. 66, p. 317-335, June 2001.

LIU, X. et al. Co-autorship networks in the digital library research community. Information Processing \& Management, v. 41, p. 1462-1480, 2005. 
LORRAIN, F; WHITE, H. C. Structural equivalence of individuals in social networks. Journal of Mathematical Sociology, v. 1, p. 49-80, 1971.

MOODY, J. The structure of a social science collaboration network: disciplinary cohesion from 1963 to 1999. American Sociological Review, v. 69, p. 213-238, Apr. 2004.

NELSON, R. Uso da análise de redes sociais no estudo das estruturas organizacionais. Revista de Administração de Empresas, v. 24, n. 4, p. 150-157, out./dez. 1984.

NEWMAN, M. E. J. Scientific collaboration networks I. Network construction and fundamental results. Physical Review E, v. 64, n. 16.131, p. 1-8, 2001a.

. Scientific collaboration networks II. Shortest paths, weighted networks and centrality. Physical Review E, v. 64, n. 16.132, p. 1-7, 2001 b.

. The structure of scientific collaboration networks. Proceedings of the National Academic Sciences, v. 98, p. 404-409, 2001c.

. Co-authorship networks and patterns of scientific collaboration. Proceedings of the National Academic Sciences, v. 101, p. 5200-5205, 2004.

ROSSONI, L.; HOCAYEN-DA-SILVA, A. J.; FERREIRA JÚNIOR, I. Estrutura de relacionamento entre instituições de pesquisa do campo de ciência e tecnologia no Brasil. Revista de Administração de Empresas, v. 48, n. 4, p. 34-48, out./dez. 2008.

SCOTT, J. Social network analysis: a handbook. 2. ed. London: Sage Publications, 2000.

SEIDMAN. S. B. Internal coheson of LS sets in graphs. Social Networks, v. 5, p. 269-287, 1983.

UZZI, B.; SPIRO, J. Collaboration and creativity: the small world problem. American Journal of Sociology, v. 111, n. 2, p. 447-504, Sept. 2005.

WAGNER, C. S.; LEYDESDORFF, L. Network strucuture, self-organization, and the growth of international collaboration in science. Research Policy, v. 34, p. 16081.618, 2005.

WASSERMAN, S.; FAUST, K. Social network analysis: methods and applications. Cambridge: Cambridge University Press, 1994.

WATTS, D. J.; STROGATZ, S. H. Collective dynamics of "small-world" networks. Nature, v. 393, p. 440-442, June 1998.

WHITE, H. C.; BOORMAN, S. A.; BREIGER, R. L. Social structure from multiple networks I. Blockmodel of roles and positions. American Journal of Sociology, v. 81, p. 730-779, 1976. 